\title{
Direct Interband Light Absorption in Conical Quantum Dot
}

\author{
D. B. Hayrapetyan, ${ }^{1,2}$ A. V. Chalyan, ${ }^{1}$ E. M. Kazaryan, ${ }^{1,2}$ and H. A. Sarkisyan ${ }^{1,2,3}$ \\ ${ }^{1}$ Russian-Armenian (Slavonic) University, H. Emin 123, 0051 Yerevan, Armenia \\ ${ }^{2}$ Yerevan State University, A. Manoogian 1, 0025 Yerevan, Armenia \\ ${ }^{3}$ Peter The Great Saint-Petersburg Polytechnic University, Polytechnicheskaya 29, St. Petersburg 195251, Russia
}

Correspondence should be addressed to D. B. Hayrapetyan; dhayrap82@gmail.com

Received 31 August 2015; Revised 3 November 2015; Accepted 4 November 2015

Academic Editor: Paulo Cesar Morais

Copyright (C) 2015 D. B. Hayrapetyan et al. This is an open access article distributed under the Creative Commons Attribution License, which permits unrestricted use, distribution, and reproduction in any medium, provided the original work is properly cited.

In the framework of the adiabatic approximation, the energy states of electron as well as the direct light absorption are investigated in conical quantum dot. Analytical expressions for particle energy spectrum are obtained. The dependence of the absorption edge on geometrical parameters of conical quantum dot is obtained. Selection rules are revealed for transitions between levels with different quantum numbers. In particular, it is shown that for the radial quantum number transitions are allowed between the levels with the same quantum numbers, and any transitions between different levels are allowed for the principal quantum number.

\section{Introduction}

The conical quantum dots (CQD) are zero-dimensional systems with nontrivial geometry, the analytic description of which is an extremely difficult problem [1-5]. From the other side, availability of many geometrical parameters characterizing the CQD allows realizing flexible manipulation of the energy levels of the charge carriers in these systems. The radius of the base and the height (or, equivalently, the cone angle) are from above-mentioned geometric parameters of CQD.

It is clear that, changing these parameters, we can manipulate the energy levels of the electrons and holes in these systems which lead to the change of the intraband and interband quantum transitions behavior. Theoretical study of physical processes in the CQD was held in [59]. It should be noted that for the theoretical description the form of confining potential of observed nanostructure has fundamental importance. In such systems, an important role may play, in particular, effects of mechanical stress. This question has been studied in $[10,11]$. Thus, the authors based on the continuum elastic theory, present a finite element analysis to investigate the influences of the elastic anisotropy and thickness of spacing layer on the strain field distribution and band edges (both conduction band and valence band) of the InAs/GaAs conical shaped quantum dots.

In another work [12], authors studied intersubband linear and third-order nonlinear optical properties of CQD with infinite barrier potential. The electron structure of CQDs through effective mass approximation is determined analytically. Linear, nonlinear, and total absorption coefficients, as well as the refractive indices of GaAs CQDs, are calculated. The effect of the dots size and the effect of the incident electromagnetic field are investigated. Results show that the total absorption coefficient and the refractive index of the dots largely depend on the size of the dots and on the intensity and polarization of the incident electromagnetic field.

As follows from the results of these studies, strain potentials are rather complicated and do not allow conduct an analytical description of the physical process at the CQD. On the other hand, the authors of papers $[5,8,9,12]$ considered the profile of the confining potential of CQD in the frame of the model of a rectangular infinitely deep well. It is noteworthy that the infinitely deep confining potential allows the partial separation of variables, and this greatly facilitates the description of CQDs. Along with the above description of the mechanisms of the CQD, in the case of a small cone angle, effective analytical method description of such systems 


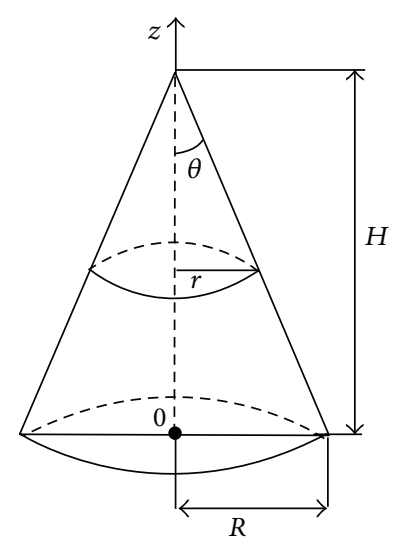

FIGURE 1: Schematic figure of conical quantum dot.

is the adiabatic approximation [13]. Under this approach, a quantum system can be represented as being linked to two subsystems: fast and slow.

The Hamiltonian of the fast subsystem includes variables as parameters of the slow subsystem, and an effective potential energy plays a slow subsystem (parametrically depending on coordinates of slow system) energy fast [14-16]. This peculiar method of separation of variables allows us to give an analytical description of single-particle states.

In this paper, the energy states of electron and direct interband light absorption in CQD are investigated within the framework of adiabatic approximation.

\section{Theory}

Let us consider an impenetrable conical QD (see Figure 1), where $H$ is height of the cone and $R$ is radius of the base of the cone. Here the angle of the top of the cone is denoted by $\theta$. Note that we consider the case of small angles of the top of cone $\theta \ll 1$. It means that $R \ll H$ and hence

$$
\operatorname{tg} \theta=\frac{R}{H} \ll 1 .
$$

Such a case is possible when, during the experiment, conical quantum dots were grown with high height [1-3]. Condition (1) gives us the opportunity to apply geometrical adiabatic approximation for solving the problem [13]. The potential energy of a charged particle (electron or hole) in such structure has the following form:

$$
U_{\text {conf }}(\rho, \varphi, z)= \begin{cases}0, & \text { particle } \in \Delta, \\ \infty, & \text { particle } \notin \Delta .\end{cases}
$$

In the regime of strong size quantization, the energy of the Coulomb interaction between the electron and hole is much less than the energy caused by the size quantization. In this approximation, the Coulomb interaction between particles can be neglected. Then, the problem is reduced to the determination of separate energy states of the electron and hole. It follows from the geometrical form of conical QD that the particle motion along the radial direction occurs more rapidly than along the $z$-direction. This allows one to use the adiabatic approximation [13]. The Hamiltonian of the system in the cylindrical coordinates has the form

$$
\begin{aligned}
\widehat{H}(\rho, \varphi, z)= & -\frac{\hbar^{2}}{2 m_{e}^{*}}\left(\frac{1}{\rho} \frac{\partial}{\partial \rho}\left(\rho \frac{\partial}{\partial \rho}\right)+\frac{1}{\rho^{2}} \frac{\partial^{2}}{\partial \varphi^{2}}+\frac{\partial^{2}}{\partial z^{2}}\right) \\
& +U_{\text {conf }}(\rho, \varphi, z)
\end{aligned}
$$

where $m_{e}^{*}$ is effective mass of charge carrier. The frequency of the radial "motion" is much greater than the frequency characterizing the "motion" along the axis of the cone. Consequently, the Hamiltonian of the system can be represented as a sum of the Hamiltonians for the "fast" $\left(\widehat{H}_{f}\right)$ and "slow" $\left(\widehat{H}_{s}\right)$ subsystems:

$$
\widehat{H}(\rho, \varphi, z)=\widehat{H}_{f}(\rho, \varphi ; z)+\widehat{H}_{s}(\rho, \varphi, z),
$$

where

$$
\begin{aligned}
\widehat{H}_{f}(\rho, \varphi ; z)= & -\frac{\hbar^{2}}{2 m_{e}^{*}}\left(\frac{1}{\rho} \frac{\partial}{\partial \rho}\left(\rho \frac{\partial}{\partial \rho}\right)+\frac{1}{\rho^{2}} \frac{\partial^{2}}{\partial \varphi^{2}}\right) \\
& +U_{\text {conf }}(\rho, \varphi, z) \\
\widehat{H}_{s}(z)= & -\frac{\hbar^{2}}{2 m_{e}^{*}} \frac{\partial^{2}}{\partial z^{2}} .
\end{aligned}
$$

Axial variable $z$ in $\widehat{H}_{f}(\rho, \varphi ; z)$ plays the role of a constant parameter. According to geometrical adiabatic approximation, the wave function of the system is represented as follows:

$$
\Psi(\rho, \varphi, z)=\frac{e^{i m \varphi}}{\sqrt{2 \pi}} f(\rho ; z) \chi(z) .
$$

By substituting wave function in the Schrödinger equation for the "fast" subsystem, for the radial wave function $f(\rho ; z)$ we get

$$
\begin{aligned}
& \frac{\hbar^{2}}{2 m_{e}^{*}}\left(f^{\prime \prime}(\rho ; z)+\frac{1}{\rho} f^{\prime}(\rho ; z)-\frac{m^{2}}{\rho^{2}} f(\rho ; z)\right) \\
& \quad+E_{\mathrm{rad}}(z) f(\rho ; z)=0
\end{aligned}
$$

where $E_{\mathrm{rad}}(z)$ is the radial part of energy. The solution of this equation is given through the Bessel functions of the first kind $J_{m}(\delta \rho)$ [17], where $\delta=\sqrt{2 m_{e}^{*} E_{\text {rad }}(z) / \hbar^{2}}$. The radial wave function should satisfy the following boundary conditions:

$$
J_{m}((H-z) \operatorname{tg} \theta)=0 .
$$

From this boundary conditions finally we obtain the expression for energy of "fast" subsystem

$$
E_{n_{\rho},|m|}^{\mathrm{rad}}(z)=\frac{\hbar^{2} \lambda_{n_{\rho},|m|}^{2}}{2 m_{e}^{*}(H-z)^{2} \operatorname{tg}^{2} \theta},
$$

where $\lambda_{n_{\rho}, m \mid}$ are zeros of the Bessel functions of the first kind $\left(n_{\rho}=0,1,2, \ldots\right)$. For the lower levels of the spectrum, the 
TABLE 1: The comparison of the energy of Coulomb interaction $\left(E_{e h}\right)$ between the electron and hole and size quantization energy $\left(E_{\mathrm{SQ}}\right)$.

\begin{tabular}{|c|c|c|c|c|c|c|c|c|}
\hline & \multicolumn{2}{|c|}{$m=0, n_{\rho}=0, n=0$} & \multicolumn{2}{|c|}{$m=1, n_{\rho}=0, n=0$} & \multicolumn{2}{|c|}{$m=0, n_{\rho}=1, n=0$} & \multicolumn{2}{|c|}{$m=0, n_{\rho}=0, n=1$} \\
\hline & $E_{\mathrm{SQ}} / E_{R}$ & $E_{e h} / E_{R}$ & $E_{\mathrm{SQ}} / E_{R}$ & $E_{e h} / E_{R}$ & $E_{\mathrm{SQ}} / E_{R}$ & $E_{e h} / E_{R}$ & $E_{\mathrm{SQ}} / E_{R}$ & $E_{e h} / E_{R}$ \\
\hline $\begin{array}{l}R=0.5 a_{B} \\
H=10 a_{B}\end{array}$ & 29.624 & -1.121 & 70.809 & -0.877 & 141.542 & -1.13 & 34.483 & -0.95 \\
\hline $\begin{array}{l}R=1.0 a_{B} \\
H=10 a_{B}\end{array}$ & 8.359 & -0.902 & 19.476 & -0.708 & 38.272 & -1.021 & 10.287 & -0.608 \\
\hline $\begin{array}{l}R=1.5 a_{B} \\
H=10 a_{B}\end{array}$ & 4.071 & -0.781 & 9.317 & -0.635 & 18.085 & -0.762 & 5.193 & -0.503 \\
\hline
\end{tabular}

particle is mainly localized in the region $|z| \ll H$. Based on this we expand $E_{n_{\rho},|m|}^{\mathrm{rad}}(z)$ into a series:

$$
E_{n_{\rho},|m|}^{\mathrm{rad}}(z)=E_{\mathrm{rad}}^{(0)}\left(1-\frac{z}{H}\right)^{-2} \approx E_{\mathrm{rad}}^{(0)}\left(1+2 \frac{z}{H}\right)
$$

where $E_{\text {rad }}^{(0)}=\hbar^{2} \lambda_{n_{\rho},|m|}^{2} / 2 m_{e}^{*} R^{2}$. Relation (10) represents an effective potential which is incorporated in Schrödinger equation of the "slow" subsystem

$$
-\frac{\hbar^{2}}{2 m_{e}^{*}} \frac{\partial^{2}}{\partial z^{2}} \chi(z)+E_{\mathrm{rad}}^{(0)}\left(1+2 \frac{z}{H}\right) \chi(z)=E \chi(z) .
$$

This equation after simple transformations reduced to the form

$$
-\frac{\hbar^{2}}{2 m_{e}^{*}} \frac{d^{2} \chi(z)}{d z^{2}}+F z \chi(z)=\left(E-E_{\mathrm{rad}}^{(0)}\right) \chi(z)
$$

where $E_{\mathrm{ax}}=E-E_{\mathrm{rad}}^{(0)}$ is the axial part of the total energy and $F=2 E_{\mathrm{rad}}^{(0)} / H$. Introducing new notations $z_{0}=\left(\hbar^{2} / 2 m_{e}^{*} F\right)^{1 / 3}$ and $\bar{z}=\left(1 / z_{0}\right)(z-E / F)$ for the Schrödinger equation of "slow" subsystem we get

$$
\frac{d^{2} \chi(\bar{z})}{d \bar{z}^{2}}-\bar{z} \chi(\bar{z})=0
$$

Solution of this equation is given by the Airy function of the first kind $\operatorname{Ai}(\bar{z})[17]$ :

$$
\chi(\bar{z})=c \operatorname{Ai}(\bar{z})=c \frac{1}{\sqrt{\pi}} \int_{0}^{\infty} \cos \left(u \bar{z}-\frac{1}{3} u^{3}\right) d u .
$$

From the boundary conditions, we get expression for the axial energy:

$$
E_{\mathrm{ax}}=-\left(\frac{\hbar^{2} F^{2}}{2 m_{e}^{*}}\right)^{1 / 3} \alpha_{n+1},
$$

where $\left\{\alpha_{n+1}\right\}$ and $(n=0,1,2, \ldots)$ are zeros of the Airy function $\left(\alpha_{1} \approx-2.338, \alpha_{2} \approx-4.087\right.$, etc.). Finally, for the total energy of the system we can write

$$
\begin{aligned}
E & =E_{\mathrm{rad}}+E_{\mathrm{ax}} \\
& =\frac{\hbar^{2} \lambda_{n_{\rho},|m|}^{2}}{2 m_{e}^{*} R^{2}}-\frac{\hbar^{2}}{2 m_{e}^{*}}\left(\frac{2 \lambda_{n_{\rho},|m|}^{2}}{H R^{2}}\right)^{2 / 3} \alpha_{n+1} .
\end{aligned}
$$

As we mentioned above in the regime of strong size quantization the energy of the Coulomb interaction between the electron and hole is much less than the energy caused by the size quantization and it can be neglected in first approximation. Table 1 presents the comparison of the energy of Coulomb interaction between the electron and hole and size quantization energy for different values of geometrical parameters of conical quantum dot. The correction energy is calculated by the help of perturbation theory. According to perturbation theory, the energy of the first correction is

$$
E_{\nu}^{1}=\langle\nu|V(\rho, z)| \nu\rangle .
$$

Here $V(\rho, z)=-e^{2} / \kappa \sqrt{\rho^{2}+z^{2}}$, where $\kappa$ is the static dielectric constant. It can be seen from Table 1 the correction energy always is negative and with the increase of radius $R$ the relative contribution of Coulomb energy of exciton becomes significant.

\section{Direct Interband Light Absorption}

Consider the direct interband absorption in conical quantum in the strong size quantization regime, when the Coulomb interaction between electron and hole can be neglected. Furthermore, consider the case of a heavy hole with $m_{e}^{*} \ll$ $m_{h}^{*}$, where $m_{e}^{*}$ and $m_{h}^{*}$ are effective masses of the electron and hole, respectively. Then, the absorption coefficient is given by [18]

$$
K(\Omega)=A \sum_{\nu, \nu^{\prime}}\left|\int \Psi_{\nu}^{e} \Psi_{\nu^{\prime}}^{h} d \vec{r}\right|^{2} \delta\left(\hbar \Omega-E_{g}-E_{\nu}^{e}-E_{\nu^{\prime}}^{h}\right),
$$

where $\Psi_{\nu\left(\nu^{\prime}\right)}^{e(h)}$ is given by expression (6), $v$ and $v^{\prime}$ are sets of quantum numbers corresponding to the electron and the heavy hole, respectively, $E_{g}$ is the band gap of massive semiconductor, $\Omega$ is the frequency of the incident light, and $A$ is a quantity proportional to the square of the matrix element taken by Bloch functions [19]. In the regime of strong size quantization for the absorption edge we finally get

$$
\hbar \Omega_{000}=E_{g}+\frac{\hbar^{2} \lambda_{0,0}^{2}}{2 \mu R^{2}}+\frac{\hbar^{2}}{2 \mu}\left(\frac{2 \lambda_{0,0}^{2}}{H R^{2}}\right)^{2 / 3} \alpha_{1},
$$

where $\mu=m_{e}^{*} m_{h}^{*} /\left(m_{e}^{*}+m_{h}^{*}\right)$ is the reduced electron-hole pair effective mass. 


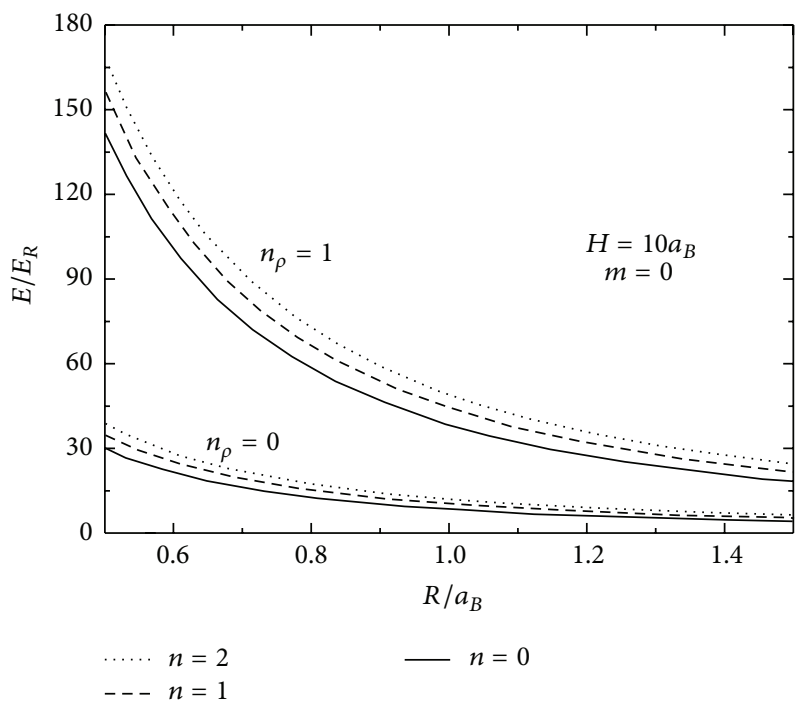

Figure 2: The dependence of the energy levels of the electron from the base radius of CQD for the fixed value of the height.

\section{Result and Discussion}

Let us proceed to the discussion of the results. Note that the numerical calculations are made for the conical QD from GaAs with the following parameters: $m_{e}^{*}=0.067 m_{e}, \kappa=$ $13.18, E_{R}=5.275 \mathrm{meV}$, and $a_{B}=104 \AA$. Figure 2 shows the dependence of the energy levels of the charge carrier from the base radius of CQD for the fixed value of the CQD's height.

Note that each level of the "fast" subsystem has a family of "slow" subsystem levels positioned thereupon. One can see from Figure 2 with increasing base radius energy of the particle reduces, since the contribution to the energy of the size quantization decreases. The difference between the energy levels of the same energy level's family is increased with increase in the axial quantum number. For example, $\Delta E_{10}=1.12 E_{R}$, when $R=1.5 a_{B}$ and $H=10 a_{B}\left(n_{\rho}=0\right.$, $m=0)$, and $\Delta E_{10}=3.4 E_{R}$, when $R=1.5 a_{B}$ and $H=10 a_{B}$ $\left(n_{\rho}=1, m=0\right)$. Note that the transition frequency between these energy levels is $\Delta \Omega_{10}\left(n_{\rho}=0, m=0\right)=1.43 \cdot 10^{12} c^{-1}$ and $\Delta \Omega_{10}\left(n_{\rho}=1, m=0\right)=4.3 \cdot 10^{12} c^{-1}$, which falls into the IR part of spectrum.

Figure 3 shows the dependence of the energy levels of the electron from the height of CQD for the fixed value of the CQD's base radius. The dependence of the energy levels on the height of CQD has the same behaviour as the dependence of radius: with the increase of the height energy levels are reduced.

Note that the total energy of the system is more "sensitive" to changes of the $R$ parameter, which is a consequence of the higher contribution of size quantization into the electron energy in radial direction. The same increase in the difference between the energy levels of the same energy level's family occurs for this dependence. For example, $\Delta E_{10}=0.58 E_{R}$, when $R=2 a_{B}$ and $H=15 a_{B}\left(n_{\rho}=0, m=0\right)$, and $\Delta E_{10}=1.76 E_{R}$, when $R=2 a_{B}$ and $H=15 a_{B}\left(n_{\rho}=1, m=0\right)$.

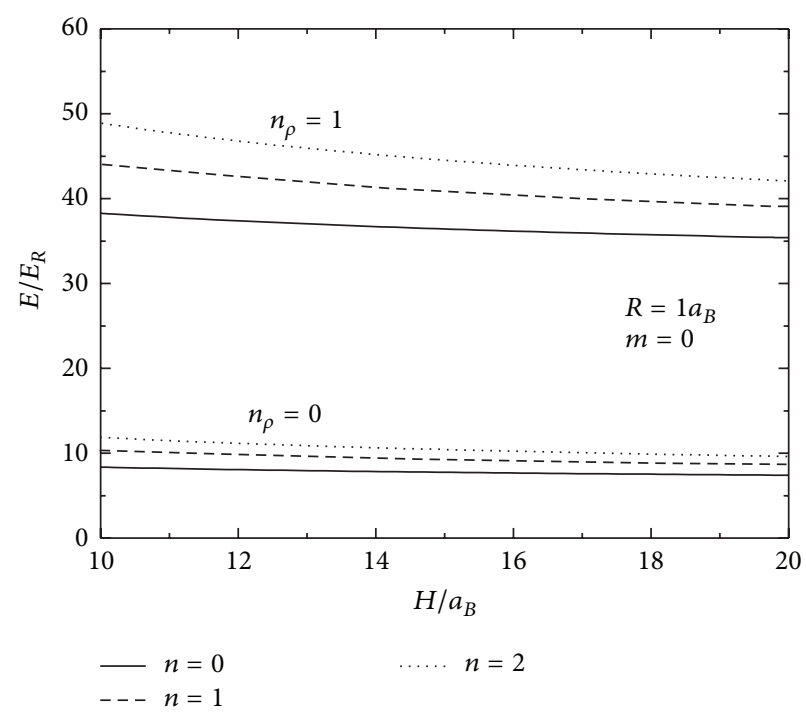

FIgURE 3: The dependence of the energy levels of the electron from the height of CQD for the fixed value of the base radius.

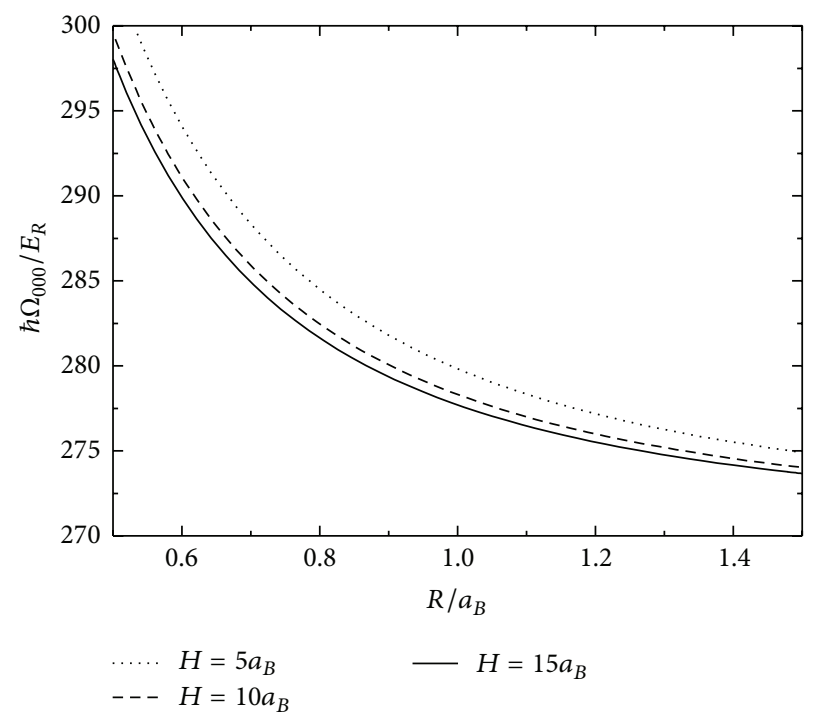

FIGURE 4: The dependence of absorption edge on the base radius of CQD for the fixed value of height.

Figure 4 shows the dependence of absorption edge on the base radius of CQD for the fixed value of height in the regime of strong size quantization. As can be seen from the figure, with decreasing base radius of CQD, the absorption edge increases. It is the consequence of the following: with the decrease of parameter $R$ the effective width of the bandgap increases by reducing the influence of the CQD's walls. The energy levels corresponding to high values of height are located above. Note that the interband transition frequency between energy levels is $\Omega_{000}=5.07 \cdot 10^{14} c^{-1}$ for $R=0.2 a_{B}$ and $H=15 a_{B}$ which falls into the visible part of spectrum.

Figure 5 shows the dependence of absorption edge on the height of CQD for the fixed value of base radius in the regime of strong size quantization. For the same reason 


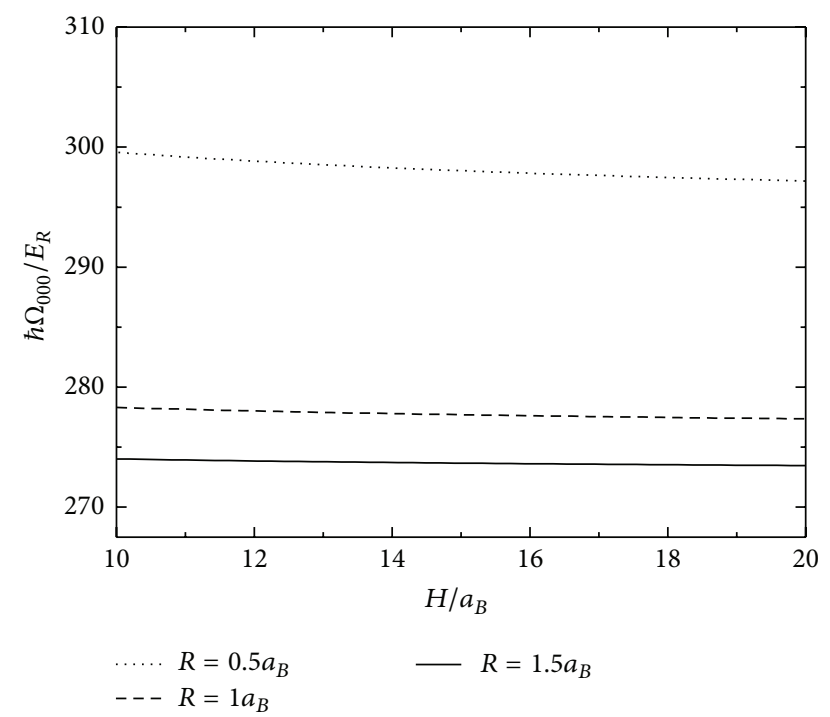

FIGURE 5: The dependence of absorption edge on the height of CQD for the fixed value of base radius.

as we mentioned for Figure 4 with increasing height of CQD, the absorption edge increases. Here the energy levels corresponding to small values of base radius are located above.

Consider selection rules for transitions between levels with different quantum numbers. For the magnetic quantum number transitions between the levels with $m=-m^{\prime}$ are allowed, and for the radial quantum number $n_{\rho}$ transitions between the levels with $n_{\rho}=n_{\rho}^{\prime}$ are allowed. Consequently, there is no selection rule for the principal quantum number and any transitions between different levels are allowed: $n \rightarrow$ $\forall n^{\prime}$.

\section{Conclusion}

Summarizing, the electronic states and optical properties of CQD made of GaAs are studied. The dependence of energy levels on the geometrical parameters of CQD is obtained analytically with the help of adiabatic approximation. Each level of the "fast" subsystem has a family of "slow" subsystem levels positioned thereupon. Note that the intraband transition frequency between energy levels falls into the IR part of spectrum, while the interband transition frequency falls into the visible part of spectrum. It is shown that for radial quantum numbers transitions are allowed between the levels with the same quantum numbers, and for the principal quantum number any transitions between different levels are allowed.

\section{Conflict of Interests}

The authors declare that there is no conflict of interests regarding the publication of this paper.

\section{References}

[1] S. Pickering, A. Kshirsagar, J. Ruzyllo, and J. Xu, "Patterned mist deposition of tri-colour CdSe/ZnS quantum dot films toward RGB LED devices," Opto-Electronics Review, vol. 20, no. 2, pp. 148-152, 2012.

[2] J.-H. Huh, C. Hermannstädter, K. Akahane et al., "Fabrication of metal embedded nano-cones for single quantum dot emission," Japanese Journal of Applied Physics, vol. 50, no. 6, Article ID 06GG02, 2011.

[3] A. Lenz, R. Timm, H. Eisele et al., "Reversed truncated cone composition distribution of $\mathrm{In}_{0.8} \mathrm{Ga}_{0.2}$ As quantum dots overgrown by an $\mathrm{In}_{0.1} \mathrm{Ga}_{0.9}$ As layer in a GaAs matrix," Applied Physics Letters, vol. 81, no. 27, pp. 5150-5152, 2002.

[4] Y. Liu, W. Lu, Z. Yu et al., "The strain field distribution of quantum dot array with conical shape," Journal of Nonlinear Optical Physics \& Materials, vol. 18, no. 4, pp. 561-571, 2009.

[5] R. Khordad and H. Bahramiyan, "Optical properties of a GaAs cone-like quantum dot: second and third-harmonic generation," Optics and Spectroscopy, vol. 117, no. 3, pp. 447-452, 2014.

[6] B. Bochorishvili and H. M. Beka, "Energy spectrum and oscillator strengths for spherical, conical and cylindrical CdSe quantum dots," IOP Conference Series: Materials Science and Engineering, vol. 6, no. 1, Article ID 012026, 2009.

[7] E. M. Kazaryan, L. S. Petrosyan, V. A. Shahnazaryan et al., "Quasi-conical quantum dot: electron states and quantum transitions," Communications in Theoretical Physics, vol. 63, no. 2, pp. 255-260, 2015.

[8] R. Khordad and H. Bahramiyan, "Study of impurity position effect in pyramid and cone like quantum dots," The European Physical Journal Applied Physics, vol. 67, no. 2, pp. 20402-20409, 2014.

[9] V. Lozovskiy and V. Pyatnytsya, "The analytical study of electronic and optical properties of pyramid -like and conelike quantum dots," Journal of Computational and Theoretical Nanoscience, vol. 8, pp. 1-9, 2011.

[10] L. Yu-Min, Y. Zhong-Yuan, and R. Xiao-Min, “The influences of thickness of spacing layer and the elastic anisotropy on the strain fields and band edges of InAs/GaAs conical shaped quantum dots," Chinese Physics B, vol. 18, no. 1, pp. 16-22, 2009.

[11] T. O. Cheche and Y.-C. Chang, "Analytical approach for strain and piezoelectric potential in conical self-assembled quantum dots," Journal of Applied Physics, vol. 104, no. 8, Article ID 083524, 2008.

[12] M. Dezhkam and A. Zakery, "Exact investigation of the electronic structure and the linear and nonlinear optical properties of conical quantum dots," Chinese Optics Letters, vol. 10, no. 12, Article ID 121901, 2012.

[13] V. Galitski, B. Karnakov, and V. Kogan, Exploring Quantum Mechanics: A Collection of 700+ Solved Problems for Students, Lecturers, and Researchers, Oxford University Press, New York, NY, USA, 2013.

[14] D. B. Hayrapetyan and E. M. Kazaryan, "Adiabatic description of impenetrable particles in an infinitely deep potential well," Journal of Contemporary Physics, vol. 47, no. 5, pp. 230-235, 2012.

[15] A. A. Gusev, O. Chuluunbaatar, S. I. Vinitsky, E. M. Kazaryan, and H. A. Sarkisyan, "The application of adiabatic method for the description of impurity states in quantum nanostructures," Journal of Physics: Conference Series, vol. 248, no. 1, Article ID 012047, 2010. 
[16] D. B. Hayrapetyan, "Direct interband light absorption in a strongly prolated ellipsoidal quantum dot," Journal of Contemporary Physics, vol. 42, no. 6, pp. 292-297, 2007.

[17] M. Abramowitz and I. Stegun, Handbook of Mathematical Functions, Applied Mathematics Series, 1966.

[18] Al. L. Efros and A. L. Efros, "Interband absorption of light in a semiconductor sphere," Semiconductors, vol. 16, no. 7, pp. 772775, 1982.

[19] A. Anselm, Introduction to Semiconductor Theory, Mir Publishers, Moscow, Russia, 1982. 

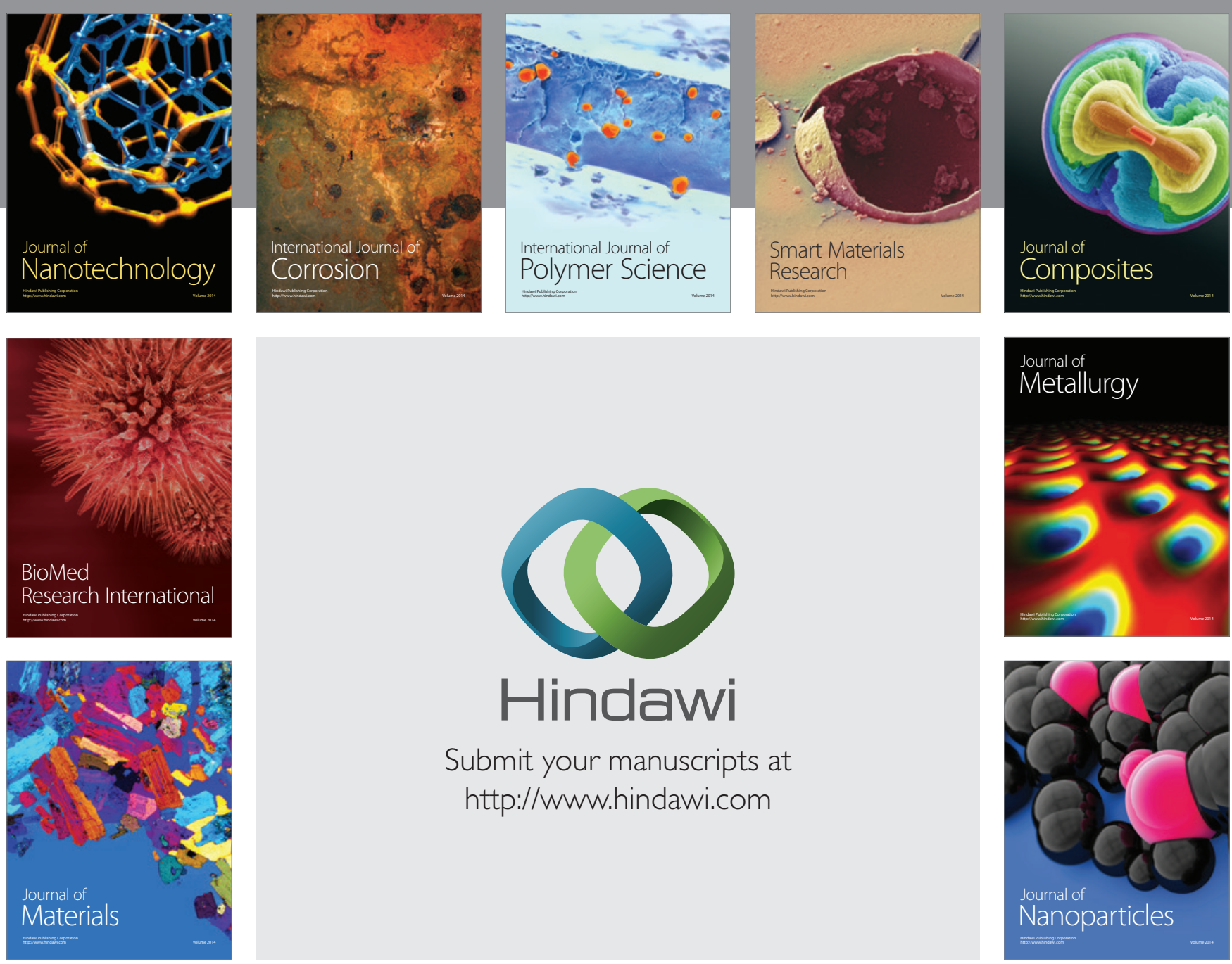

Submit your manuscripts at http://www.hindawi.com
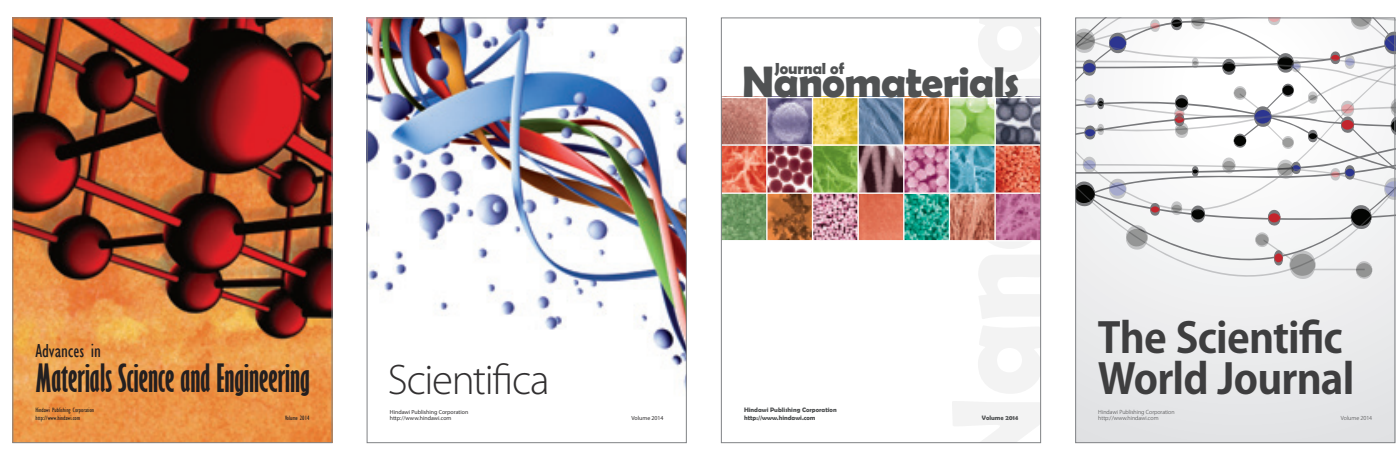

\section{The Scientific World Journal}
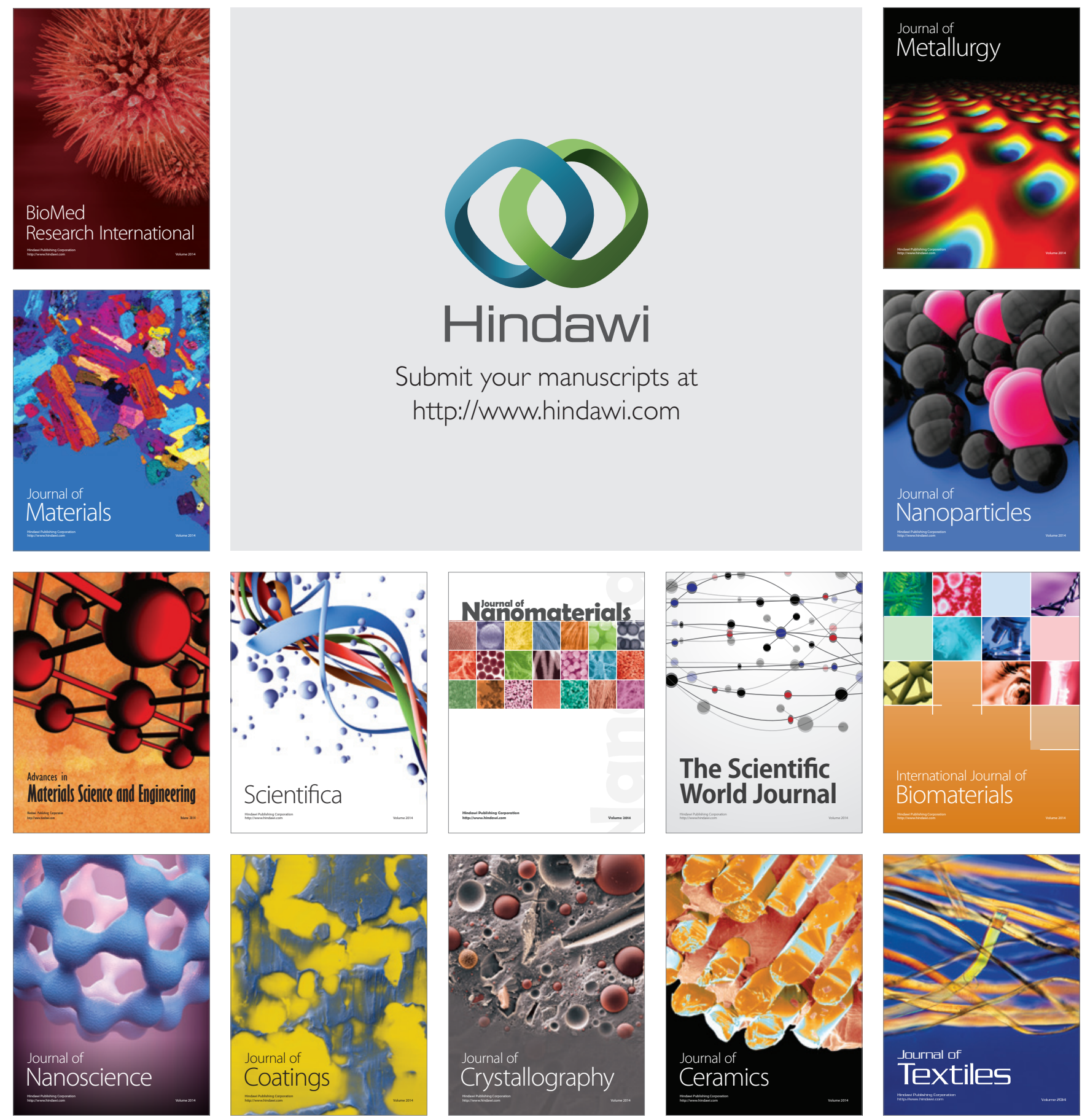